\title{
Aplicação do processamento de imagens por computador na quantificação das variáveis histopatológicas da reparação tecidual de anastomoses colocólicas em cães
}

\author{
M.D. Novelli, ${ }^{*}$ E. Barreto, ${ }^{* *}$ D. Matos, ${ }^{*}$ S.S. SaAd, R.C. Borra \\ Disciplina de Patologia Geral, Departamento de Estomatologia da Faculdade de Odontologia da Universidade de São \\ Paulo.*Serviço de Anatomia Patológica do Hospital do Servidor Público Estadual de São Paulo. **Departamento de Cirurgia da \\ Universidade Federal de São Paulo - Escola Paulista de M edicina, São Paulo, SP.
}

\begin{abstract}
RESUMO - OвJ ETIvo. Os autores apresentam a metodologia de quantificação, através do processamento de imagens, de estruturas teciduais microscópicas envolvidas no processo de reparação de anastomoses colocólicas, feitas por meio de sutura manual e por anel biofragmentável. As variáveis quantificadas neste trabalho foram líquido de edema, tecido fibromuscular, vasos e núcleos celulares.

Material e Métodos. Para esta análise, foi utilizado programa de processamento de imagens desenvolvido no Laboratório de Informática Dedicado à Odontologia (LIDO) da Universidade de São Paulo, com a finalidade de quantificar as alterações patognomônicas do processo inflamatório em anastomoses colocóli cas em 14 cães. Os resultados foram comparados, como contraprova, aos obtidos através de leituras convencionais feitas
\end{abstract}

\section{NTRODUÇÃO}

O campo da computação gráfica tem si do pesquisado por grande número de especialistas, dadas as suas possibilidades reais de aplicação nas descobertas científicas. Envolve todo e qualquer tipo de tratamento de imagens, desde simples gráficos até imagens complexas de projetos arquitetônicos.

Filgueras et al. ${ }^{1}$ apresentam a defini ção de computação gráfica como um conjunto de métodos e técnicas para conversão de dados para di spositivos gráficos, através de computador, adotada pela International Standard Organization (ISO). É também dos autores a classificação da computação gráfica que explica o tratamento da imagem como sendo "a síntese, a análise e o processamento de imagem", que será adotada nesta metodologia.

A aplicação do tratamento de imagens por computador tem suas origens em trabalhos escassos desde a década de 70 . Com relação ao processamento de imagens em microscopia óptica, Russ ${ }^{2,3}$ pode ser considerado o catalisador da aplicação por dois patologistas. A gradação de alterações ausentes, leves, moderadas e intensas foi utilizada nesta leitura para que se semiquantificassem as mesmas variáveis lidas pelo computador.

Resultados. Houve diferença significativa entre as duas técnicas, com a de anel biofragmentável apresentando menor quantidade de edema, fibras organizadas e prevalência de núcleos celulares alongados em relação aos esféricos, sinal evidente de organização tecidual.

Conclusão. Os resultados permitiram concluir que a histometria por processamento de imagem computacional mostrou-se eficiente na quantificação das principais estruturas teciduais envolvidas no processo inflamatório e reparativo.

UNITERMOS: Processamento de imagens por computador. Computação gráfica. Anastomose intestinal.

desses métodos, devido a sua iniciativa de editar uma revista especializada, apresentando cinco trabal hos de sua autoria logo no primeiro número.

Assim como o trabal ho de Russ, a apresentação de model os matemáticos com o intuito de "modelar" imagens tem sido a proposta de vários autores que trabalham nesse campo da computação gráfica e que a aplicam em diver sas áreas. Entretanto, foi impossível encontrar na literatura a apresentação de um produto final aberto, ou seja, que permita um exame do software e de eventuais modifi cações em função das necessidades dos pesquisadores. As publicações que serviram de base para o presente trabal ho foram uma revisão sobre o assunto, realizada por Barros \& Araújo ${ }^{4}$, e uma introdução ao processamento digital de imagens, apresentada por Mascarenhas et al. ${ }^{5}$ Cabe dizer que a escassez de publicações não reflete, contudo, as pesquisas feitas neste campo de conhecimento e, sim, a importância do sigilo comercial, pelo menos enquanto se caracterizarem como tecnologia de ponta. 


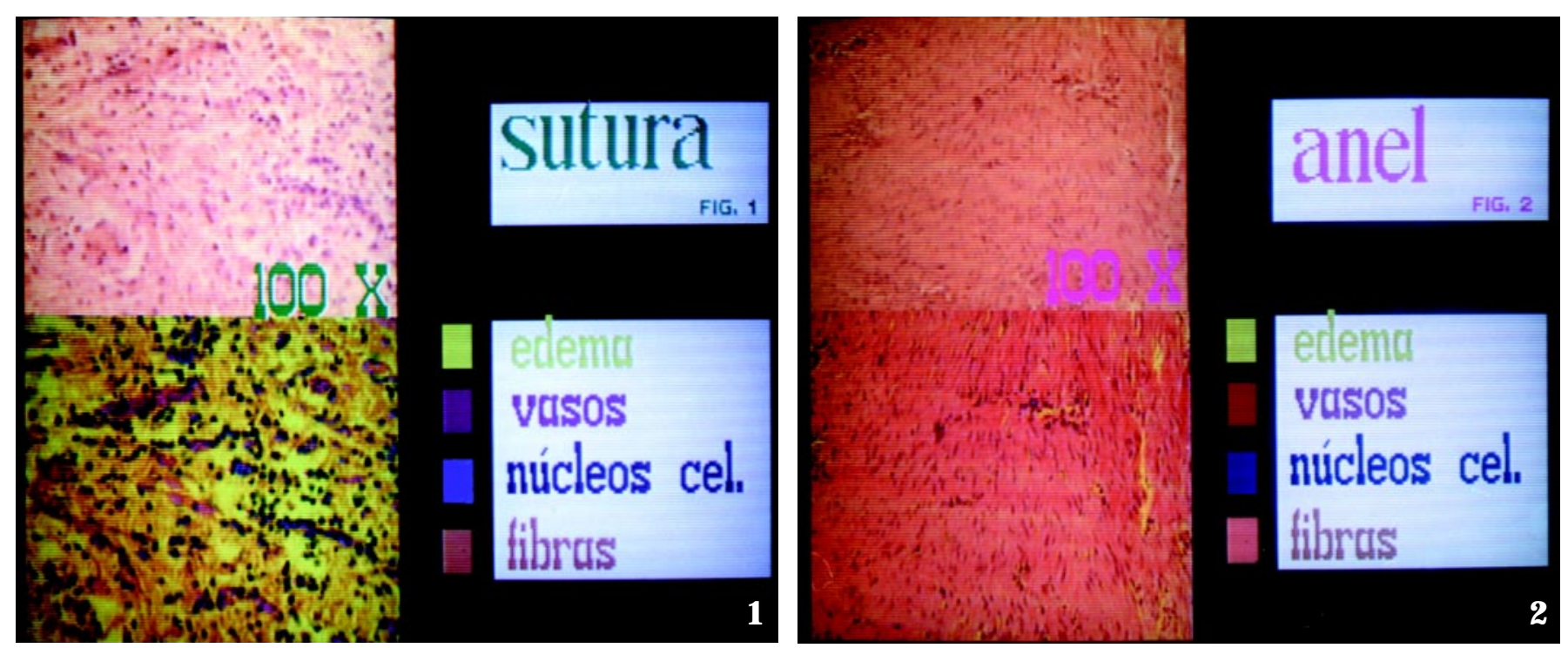

Figs. 1 e 2 - Comparação dos cortes histológicos entre as técnicas com sutura manual e com anel bi ofragmentável e as relações entre estruturas analisadas. Na imagem histológica superior de ambas as técnicas, observam-se o aspecto usual do corte e, na inferior, a mesma imagem após o destaque das estruturas através do processamento com auxílio do computador.
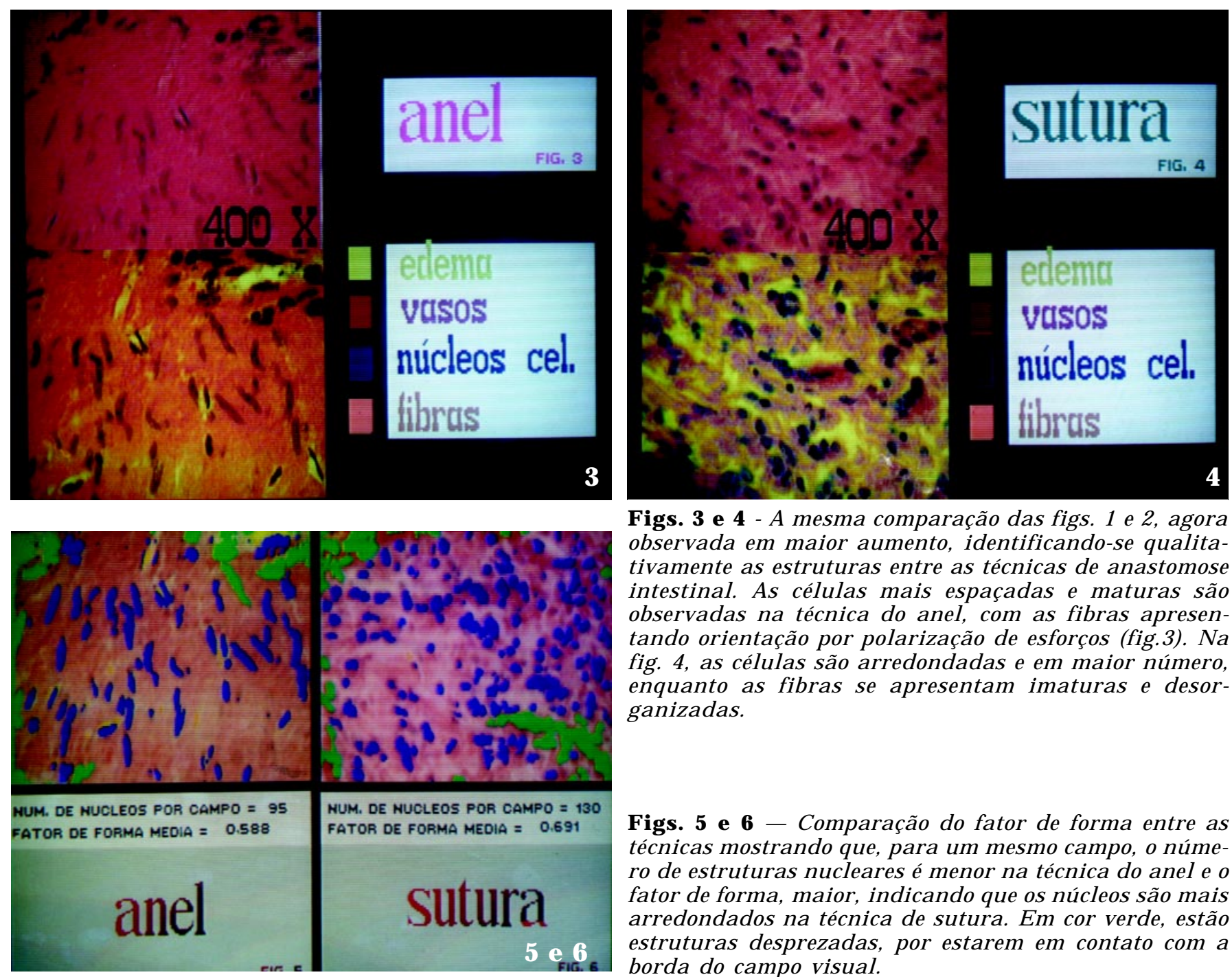

Figs. 3 e 4 - A mesma comparação das figs. 1 e 2, agora observada em maior aumento, identificando-se qualitativamente as estruturas entre as técnicas de anastomose intestinal. As células mais espaçadas e maturas são observadas na técnica do anel, com as fibras apresentando orientação por polarização de esforços (fig.3). Na fig. 4, as células são arredondadas e em maior número, enquanto as fibras se apresentam imaturas e desorganizadas.

Figs. 5 e 6 - Comparação do fator de forma entre as técnicas mostrando que, para um mesmo campo, o número de estruturas nucleares é menor na técnica do anel e o fator de forma, mai or, indicando que os núcl eos são mais arredondados na técnica de sutura. Em cor verde, estão estruturas desprezadas, por estarem em contato com a borda do campo visual. 
O processamento de imagens microscópicas por computador, especialmente com relação ao experimento com anastomoses colocólicas em cães, foi aplicado no trabalho de Matos et al. ${ }^{6} \mathrm{~N}$ essa pesquisa, foi feito um estudo comparativo de duas técnicas de anastomose, com sutura manual e com anel biofragmentável, através de uma metodologia conveniente para a interpretação de dados comparativos em que se quantificou o líquido de edema presente nos cortes histológicos de cada uma das técnicas. Essa característica serviu de base para que esse trabalho fosse tido como parâmetro para o presente estudo.

O objetivo deste trabal ho é, pois, apresentar a metodologia de processamento de imagens microscópicas em patologia experimental, quantificando as diferentes estruturas relacionadas com os processos inflamatórios envolvidos com a reparação de anastomoses colocólicas em cães.

\section{MATERIAL E MÉTODO}

Foram utilizados, neste estudo, cortes histológicos obtidos de 28 anastomoses realizadas em 14 cães, com as biópsias feitas, em média, 33 dias após a cirurgia. Quatorze anastomoses foram feitas pela técnica de sutura manual, seromuscular, pontos separados, com fio monofilamentar Maxon 000, e quatorze pela técnica com anel biofragmentável - Valtrac. Todas operações foram realizadas por um mesmo cirurgião e equipe cirúrgica, no Laboratório de Cirurgia Experimental do Hospital do Servidor Público Estadual de São Paulo.

Os cortes histológicos obtidos das áreas das anastomoses foram corados em $\mathrm{H}$-E e analisados através do software DIRACOM3, desenvolvido no Laboratório de Informática Dedicado à Odontologia (LIDO) da Faculdade de Odontologia da Universidade de São Paulo.

Esse software, concebido em linguagem $C$ por intermédio do Turbo C (Borland), permite identificar, selecionar e subtrair estruturas de uma imagem através do espectro de $32 \times 1.000$ tonalidades de cores. Define os padrões morfológicos através da área e do perímetro para o cálculo do fator de forma, que é obtido da relação dessas duas variáveis.

Para se ter esses valores de área, perímetro e fator de forma, há a separação de estruturas de imagem através da identificação e subtração de um determinado ponto da tela, denominado "pixel", com o respectivo valor de cor no intervalo entre $0 \mathrm{e}$ 32 tons. Para tal, sel eciona-se, através de um quadrado de tamanho variável, uma amostragem da imagem. Todos os valores das cores dos "pixels" no interior do quadrado são guardados em uma tabela de padrões. Se o val or da cor do "pixel" se repetir n vezes, apenas um valor será armazenado no interior do quadrado. Após esse procedimento, o computador varre toda a imagem, comparando o valor da cor de cada "pixel" com a tabela de padrões. Se o valor corresponder a algum outro presente na tabela, este é subtraído da imagem, de tal forma que permanecem somente as estruturas formadas pelos "pixels" diferentes daqueles pertencentes ao padrão selecionado. Outro padrão, após cada varredura, pode ser novamente determinado efetuandose o mesmo procedimento de posicionamento do quadrado em outra área da imagem. Assim, definese o momento adequado da subtração.

Ao final, o computador informa a percentagem das áreas removidas em rel ação à imagem original .

Cada corte histológico obti do das duas formas de anastomose passou por esse software, com a digitalização da imagem microscópica e a separação, pel o método descrito acima, das estruturas básicas que compõem os tecidos, identificadas como líquidos intersticiais, cadeias protéicas organizadas em fibras colágenas e musculares, vasos e estruturas nucleares de células. As estruturas celulares foram identificadas através da morfologia nuclear e os linfócitos mononucleares foram agrupados para serem utilizados como indicadores representativos da atividade proliferativa.

Estabelecidas as tabelas de padrões dessas estruturas, conforme o descrito anteriormente, passou-se a quantificá-las em aumentos de 100 e 400 vezes, para precisar os aspectos quantitativos e qualitativos e suas respectivas relações percentuais. Deve-se enfatizar que todas as estruturas observadas nas imagens puderam ser quantificadas, sendo expressas em valores percentuais.

E sses resultados foram comparados com os obtidos pelos métodos clássicos de semiquantificação subjetiva feita por dois patologistas que, independentemente, examinaram os mesmos cortes histológicos e classificaram as estruturas observadas por intermédio de uma gradação de val ores correspondentes às alterações ausentes, leves, moderadas e intensas.

\section{RESULTADOS}

As figs. 1 e 2 mostram, por meio da comparação entre a anastomose feita com sutura manual e a feita com anel biofragmentável e entre os seus respectivos cortes, diferença significativa entre as duas técnicas: a técnica com anel apresentava menor quantidade de edema, fibras organizadas e prevalência de núcleos celulares alongados em 
relação aos esféricos, sinal evidente de organização tecidual.

As figs. 3 e 4 mostram a mesma diferença relati va entre as estruturas que compõem a imagem, levando-se em consideração, nesse caso, os aspectos morfológicos.

As figs. 5 e 6 comparam as relações de área e de perímetro dos núcleos, corados em azul, das células envolvidas no processo de reparação. O fator de forma médio das estruturas completa a análise morfométrica, estabel ecendo uma nítida diferença entre as formas nucleares envolvidas.

A tabela ao lado é a representação gráfica comparativa dessa variável (fator de forma), traduzindo em percentagens as estruturas presentes em cada técnica analisada. Nessa tabela, os valores obtidos das médias das áreas expressam as variações entre estruturas lato sensu e "não-estruturas" (qualquer estrutura não referenciada pelos padrões de análise histométrica convencionais que foram subtraídas no processamento de imagem). Cabe lembrar que a análise dessa variável é suficiente para demonstrar a variação entre as técnicas, conforme mostra a tabela. O valor percentual na técnica com anel é muito próximo do tecido normal, ao passo que, na técnica de sutura, é praticamente o dobro.

\section{DISCUSSÃO}

O procedimento inicial no tratamento da imagem, como já foi dito, é a sua seleção, por amostragem, em um pequeno retângulo e a subtração das estruturas de interesse por intermédio dos espectros R (red), G (green) e B (blue).

Atingindo o nível satisfatório de escolha, a subtração dessas estruturas é estendida para todo o campo. Assim, se a estrutura selecionada for, por exemplo, o núcleo das células, estes serão subtraídos, deixando em seu lugar um fundo preto.

Esse é um momento críti co do sistema, uma vez que a subtração de estruturas é feita por critérios subjetivos; no caso da subtração dos núcleos das células, se o limite desses núcleos for impreciso, introduzem-se pequenos erros na leitura final, tanto da área como do perímetro. Para contornar esta dificuldade, deve-se buscar o equilíbrio entre o quanto aumentou a resolução da objetiva do microscópio e o número de estruturas a serem contadas, que necessariamente seguem uma relação inversa; quanto maior for o aumento, menor o número de estruturas a serem contadas, mas, em contrapartida, o seu contorno será melhor definido. Entretanto, quanto maior o número de estruturas, maior é a tendência a se apresentarem
Tabela - Valores obtidos da análise histológica do trato intestinal de 14 cães submetidos a duas técnicas de anastomose colocólica (anel biofragmentável e sutura manual), comparando-se com o tecido normal e distante da anastomose no mesmo animal

\begin{tabular}{|lccc|}
\hline \multicolumn{1}{|c}{ Estruturas } & $\begin{array}{c}\text { Sutura } \\
\text { manual }\end{array}$ & $\begin{array}{c}\text { Anel } \\
\text { biofragmentável }\end{array}$ & $\begin{array}{c}\text { Tecido } \\
\text { normal }\end{array}$ \\
$\begin{array}{l}\text { Núcleos } \\
\text { Líquido intersticial }\end{array}$ & $23 \%$ & $11 \%$ & $14 \%$ \\
$\begin{array}{l}\text { (edema) } \\
\begin{array}{l}\text { Fibras colágenas } \\
\text { e musculares }\end{array}\end{array}$ & $57 \%$ & $44 \%$ & $12 \%$ \\
$\begin{array}{l}\text { Total } \\
\text { Variação médias das }\end{array}$ & $100 \%$ & $45 \%$ & $74 \%$ \\
áreas “não-estruturas" & $8,96 \%$ & $100 \%$ & $100 \%$ \\
\hline
\end{tabular}

puntiformes, conseqüentemente, menor definição de seus contornos.

As pequenas variações de leitura, na verdade, poderão não constituir propriamente um erro, principal mente se o número de regiões amostradas (subtraídas) for superior a 160. Esse fato é ainda atenuado por serem leituras que só têm significado quando comparadas. Como o sistema de censoreamento é o mesmo, eventuais erros de amostragem se anulam por equiparação.

Uma característica muito importante desse software é a possibilidade de quantificar também estruturas não sel ecionadas. Em um campo microscópico, se subtrairmos os núcleos e os espaços intersticiais, ficaremos apenas com o citoplasma dessas células, passíveis de serem quantificados e relacionados com as estruturas subtraídas, que também são quantificáveis. O resultado apresentado em percentagem é semel hante ao processo de quantificação relativa de estruturas por centrifugação que, nesse caso, é a quantificação el etrônica da energia cromática. Essa possibilidade de quantificação de estruturas, em termos de sua rel atividade com a estrutura imediatamente vizinha, pode ser a primeira evidência para a aplicação prática das singularidades de Witney (\%) na determinação dos contornos suaves de estruturas biológicas teciduais.

Quantificar essas unidades relativamente aos seus universos é o ponto rel evante deste trabal ho. Os demais cálculos e fundamentos disponíveis nesse programa são conseqüência desta visão métrica das estruturas estudadas, em que o denominador comum é a análise de pontos vizinhos e dos vizinhos dos vizinhos. Assim, se um ponto é azul e seus vizinhos são azuis, estamos no universo núcleo. O mesmo raciocínio vale para o rosa, portanto, universo citoplasma. Entretanto, se o vizinho do ponto verde for o rosa, estamos na transição da forma. 
Com relação a essa transição da forma, quanto maior for o número de tons de azul, verde ou vermel ho e suas combinações, maiores serão as probabilidades de separar estruturas dentro dos seus limites. Neste sentido, o software DIRACOM 3 está limitado a 32 tons de vermel ho, 32 de azul e 32 de verde, em um total de $32 \times 1.000$ tons.

Essa metodol ogia de processamento de imagens microscópicas por computador foi aplicada, inicialmente, na quantificação de edema nas anastomoses colocólicas em cães ${ }^{6}$, em decorrência da certeza dos parâmetros clínicos identificados e comprovados após a segunda laparotomia. A análise macroscópica dos achados da segunda cirurgia mostrou nível de reparação satisfatório em ambas técnicas. Entretanto, na análise microscópica, observam-se indícios de líquido de edema intersticial em mai or quantidade nas anastomoses com sutura manual. Baseando-se nesses achados, passou-se ao estudo das demais variáveis teciduais envolvidas no processo de reparação, relatadas neste trabalho.

Com os recursos da computação gráfica, pudemos identificar e separar estruturas que até então eram difíceis de serem quantificadas em preparados histológicos, como líquido de edema, fibras musculares, fibras colágenas hialinizadas ou não e substâncias amorfas. Já as estruturas bem definidas, como núcleos celulares e vasos sanguíneos, eram passíveis de mensuração pelas técnicas histométricas clássicas. Com o processamento de imagem, todas essas variáveis histológicas puderam ser quantificadas, medidas e classificadas em padrões, conforme foram apresentas na tabela.

Como contraprova do método, executou-se a análise das mesmas variáveis pelos métodos clássi cos de avaliação subjetiva, usando a cl assificação de alterações ausentes, leves, moderadas e intensas. Constatamos que os valores obtidos pelos métodos clássicos de quantificação eram próximos aos concluídos pela análise computacional, mostrando diferenças estatísticas significantes entre as duas técnicas de anastomoses.

Os métodos de análise qualitativa e quantitativa em histopatologia por computador têm-se mostrado eficientes e rápi dos na análise de variáveis conhecidas e bem definidas. Entretanto, a quantificação de estruturas de contorno e textura imprecisos de variáveis histopatológicas foi o grande desafio deste trabal ho, dando origem a um novo enfoque na interpretação dos achados microscópicos, classificando-se em padrões bem definidos e ilimitados.

U m outro aspecto do processamento de imagem por computador refere-se à possibilidade de se anal isar todos os parâmetros mencionados - área, perímetro e fator de forma - a partir das posições ortogonais dos pontos na tela do computador (Iinhas e colunas), criando combinações que permitam discriminar val ores conspícuos de interesse não só para a histometria, mas, sobretudo, para dar subsídios à inteligência artificial.

\section{CONCLUSÕES}

O método de análise de variáveis histométricas por processamento de imagens computacional mostrou-se eficiente e poderoso na quantificação das principais variáveis teciduais envolvidas em processos inflamatórios e reparativos.

Foram passíveis de quantificação variáveis até então não processáveis e definidas como o contorno limite deixado pela subtração das estruturas convencionais, tais como células, núcl eos e vasos. Permite-se, dessa forma, quantificar em microscopia óptica áreas ocupadas por líqui do de edema, fibras colágenas e musculares, bem como substâncias intersticiais amorfas.

\section{SUMMARY}

\section{Computer-assisted image processing for quanti- fying histopathologic variables in the healing of colonic anastomosis in dogs}

BACKGROUND. The authors present the experimental results of the computerized quantifying of tissular structures involved in the reparative process of colonic anastomosis performed by manual suture and bi ofragmentable ring. The quantified variables in this study were: oedema fluid, myofiber tissue, blood vessel and celular nuclei.

Material and Methods. An image processing software devel oped at Laboratório de I nformática Dedicado à Odontologia (LIDO) was utilized to quantifying the pathognomonic alterations in the inflammatory process in colonic anastomosis performed in 14 dogs. The results were compared to those obtained through traditional way diagnosis by two pathologists in view of counterproof measures. The criteria for these diagnoses were defined in levels represented by absent, light, moderate and intensive which were compared to analysis performed by the computer.

RESULTS. There was significant statistical difference between two techniques: the bi ofragmentable ring technique exhibited low oedema fluid, organized myofiber tissue and higher number of alongated celular nuclei in relation to manual suture technique. 
Conclusion. The analysis of histometric variables through computational image processing was considered efficient and powerful to quantify the main tissular inflammatory and reparative changing. [Rev Ass Med Brasil 1997; 43(4): 277-82.]

KEY WORDS: Computer-assisted image processing. Computer graphics. Intestinal anastomosis.

\section{REFERÊNCIAS BIBLIOGRÁFICAS}

1. Filgueiras LVL, Tori R, Massola Ama, Arakaki R. Fundamentos decomputaçãográfica. Rio deJ aneiro, SãoPaulo, LTC
- Livros Técnicos e Científicos Editora, 1987.

2. Russ J C. Automatic methods for the measurement of curvature of lines, features and feature alignment in images. J Comput Assist Microsc 1989; 1: 39-77.

3. Russ J C. Computerized object recognition using contextual learning. J Comput Assist Microsc 1989; 1: 106-9.

4. Barros MA, Araújo AA. Suavização espacial: uma revisão. In: J ornada EPUSP/IEEE em Computação Visual. Anais, São Paulo,1990; 454.

5. Mascarenhas NDA. Introdução ao processamento digital de imagens. Anais, São Paulo, 1990; 387-420.

6. Matos, D, Saad SS, Franceschi J r O, Barreto E, Novelli MD. Estudo experimental comparativo entre anastomose colocólica com sutura manual e com anel biofragmentável. Rev Ass Med Brasil 1993; 39: 201-6. 